\title{
The effect of laser wavelength on heating of ablated carbon plume
}

\author{
J. Hoffman · T. Moscicki · Z. Szymanski
}

Received: 3 August 2010 / Accepted: 8 April 2011 / Published online: 7 May 2011

(c) The Author(s) 2011. This article is published with open access at Springerlink.com

\begin{abstract}
The effect of laser wavelength on heating of the ablated carbon plume is studied. The plasma absorption coefficients are calculated in order to analyze the results and an experiment is conducted using the first, second, and third harmonic of a Nd:YAG laser. Plasma temperature and electron density in the early phase of expansion in vacuum are studied as a function of distance from the target. The calculations show that the ratio between absorption coefficients for 1064,532 , and $355 \mathrm{~nm}$ is approximately 9:2:1. The experimental results do not agree well with the theoretical predictions. Indeed, the plasma temperatures are highest in the case of $1064 \mathrm{~nm}$ but no clear differences between $532 \mathrm{~nm}$ and $355 \mathrm{~nm}$ have been found.
\end{abstract}

\section{Introduction}

During laser ablation, the material evaporated from the target forms a thin layer of very dense gas, consisting of electrons, ions, and neutrals. This plasma plume absorbs energy from the laser beam and its temperature and pressure grow. Main heating mechanisms are inverse Bremsstrahlung and photoionization and both mechanisms depend on laser wavelength. While electron-ion inverse Bremsstrahlung is easy to calculate since Coulomb cross section is well known, the electron-atom inverse Bremsstrahlung and photoionization require the knowledge of electron-atom scattering and photoionization cross sections.

J. Hoffman · T. Moscicki · Z. Szymanski (凶) Institute of Fundamental Technological Research, Pawinskiego 5B, 02-106 Warsaw, Poland

e-mail: zszym@ippt.gov.pl

Fax: +48-22-8267380
In this paper, the effect of laser wavelength on heating of ablated carbon plume is studied. Graphite was chosen because its ablation is used to obtain a wide variety of carbonrelated materials, such as diamond-like carbon, fullerenes, and carbon nanotubes. Moreover, the relevant cross sections for carbon atoms and ions are available which enables the analysis of the photon absorption. Although temperatures of plasmas induced during carbon ablation with the use of different lasers were studied in several papers [1-6], the comparison of results obtained in [1-6] is inconclusive because laser intensities, distances from the target and delay times differed considerably. Therefore, in this paper, all relevant parameters are kept constant what enables the comparison and analysis of the results obtained for different laser wavelengths.

\section{Photon absorption}

The laser beam interacting with the plasma heats the electrons by two mechanisms; the inverse Bremsstrahlung (IB) and photoionization (PI). The heating term is $\kappa \cdot I$, where $\kappa$ is the absorption coefficient and $I$ is a laser intensity.

The total absorption coefficient can be written as

$$
\begin{aligned}
\kappa_{\text {total }}= & \left(\kappa_{\mathrm{ei}}^{\mathrm{IB}}(\lambda)+\kappa_{\mathrm{ea}}^{\mathrm{IB}}(\lambda)\right. \\
& \left.+\kappa^{\mathrm{PI}}(\lambda)\right) \times\left[1-\exp \left(-\frac{h c}{\lambda k T_{e}}\right)\right]
\end{aligned}
$$

where indices "ei" and "ea" denote electron-ion and electronatom collisions. The last bracket contains contribution from the stimulated emission. Formulas for $\kappa_{\mathrm{ei}}^{\mathrm{IB}}$ and $\kappa^{\mathrm{PI}}$ were taken from [7] and for $\kappa_{\mathrm{ea}}^{\mathrm{IB}}$ from [8] and are written below 
Fig. $1 \xi$-Factors for carbon
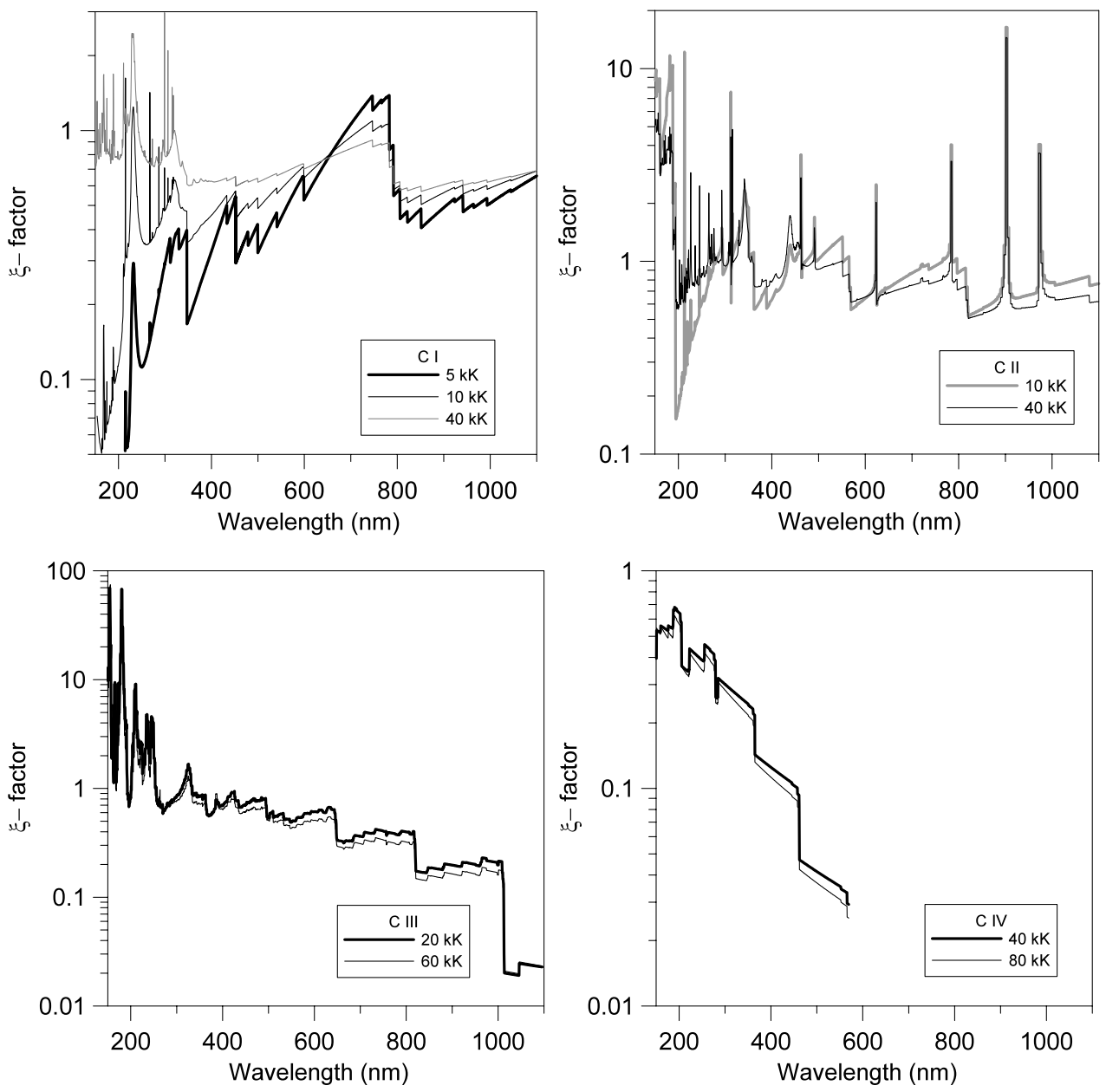

(in SI units)

$$
\begin{aligned}
\kappa^{\mathrm{PI}}(\lambda)= & 6.6 \times 10^{-6} T_{e} \lambda^{3} \frac{g_{z, 1}}{U_{z-1}} \sum_{z} z^{2} n_{z-1} \\
& \times \exp \left\{-\frac{E_{j}}{k T_{e}}\right\}\left(\exp \left\{\frac{h c}{\lambda k T_{e}}\right\}-1\right) \xi\left(T_{e}, \lambda\right) \\
\kappa_{\mathrm{ei}}^{\mathrm{IB}}(\lambda)= & 1.3674 \times 10^{-27} \lambda^{3} n_{e} T_{e}^{-1 / 2} \sum_{z=1} z^{2} n_{z} G
\end{aligned}
$$$$
\kappa_{\mathrm{ea}}^{\mathrm{IB}}(\lambda)=8.6 \times 10^{-19} \lambda^{3} n_{a} n_{e} T_{e}^{3 / 2} \sigma_{e a}\left(T_{e}\right)
$$$$
\times\left[1+\left(1+\frac{h c}{\lambda k T_{e}}\right)^{2}\right]
$$

where $n_{z-1}$ is the density of parent atom or ion $\left(n_{0} \equiv n_{a}\right)$, $n_{e}$ and $n_{z}$ are the electron and ion density, $T_{e}$ is the electron temperature, $z$ denotes ion charge seen by free electron, $U_{z-1}$ is the partition function, $E_{j}$ is the ionization energy, $g_{z, 1}$ is the statistical weight of the ground state of the ion, $G$ is the Gaunt factor [9], $\sigma_{\text {ea }}$ is the electron-atom scattering cross section, and $\xi$ is a correction factor [7], which introduces the edge structure of the real coefficients.
By definition $\xi\left(T_{e}, \lambda\right)=\kappa^{\mathrm{PI}}(\lambda) / \kappa^{\mathrm{PI}, H}(\lambda)$, where $\kappa^{\mathrm{PI}}(\lambda)=\sum_{E_{i} \leq E_{\mathrm{ph}}} n_{z-1, i} \sigma_{z-1, i}(\lambda, T)$ (the summation is over all levels with the ionization energy lower than the photon energy) and $\kappa^{\mathrm{PI}, H}(\lambda)$ is the absorption coefficient given by (2) with $\xi=1$. The $\xi$-factors for carbon were calculated with the use of photoionization cross section from [10] assuming the local thermodynamic equilibrium (LTE). The results are shown in Fig. 1. The spikes seen in the figures result from the photo-ionization with simultaneous excitation of the arising ion and transitions to auto-ionized states. The electron-atom absorption coefficient based on the cross section calculated in [11] is shown in Fig. 2.

In the case of nanosecond laser pulse, the ablation is thermal. The particles released from the target reach Maxwellian distribution after some collisions in the Knudsen layer [12]. The vapour density $\rho_{v}$, the pressure $p_{v}$, and the temperature $T_{v}$ at the end of the Knudsen layer depend on the Mach number only [12]. If $M=1$, then $T_{v} \sim 0.67 T_{s}, p_{v} \sim 0.21 p_{s}$, $\rho_{v} \sim 0.31 \rho_{s}$ [12], where the subscript $s$ denotes the surface. The temperature $T_{s}$ is determined solving heat transfer equation and the pressure $p_{s}$ is calculated from the ClausiusClapeyron equation. 


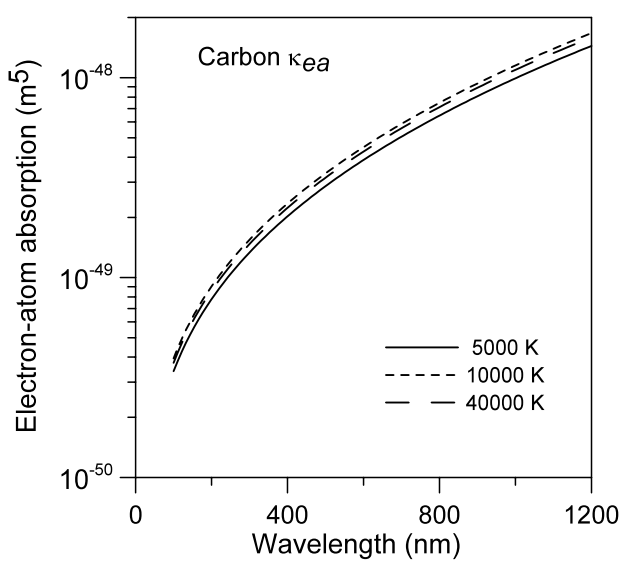

Fig. 2 Electron-atom absorption coefficient per unit electron and atom density

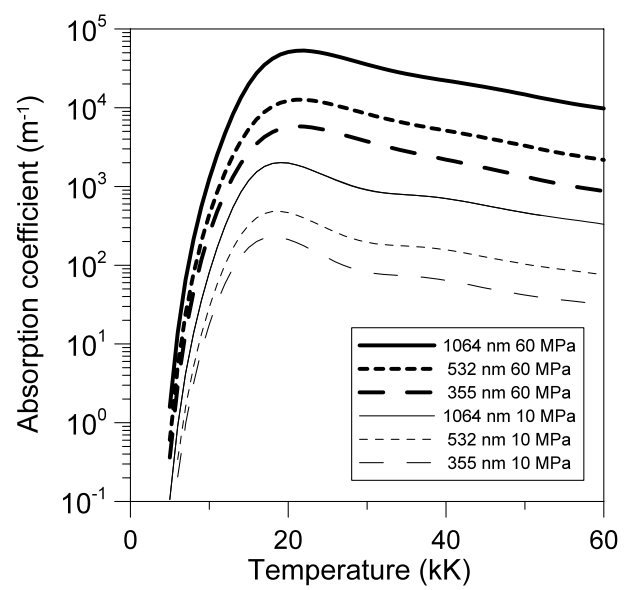

Fig. 3 Plasma absorption coefficients for various laser wavelengths as a function of temperature

The maximum temperature of graphite target in the case of $1064 \mathrm{~nm}$ laser wavelength is about $7800 \mathrm{~K}$ for laser fluence $F=15 \mathrm{~J} \mathrm{~cm}^{-2}$ [13]. At this temperature the saturated vapour pressure is about $285 \mathrm{MPa}$, and hence $p_{v}=60 \mathrm{MPa}$, $T_{v}=5200 \mathrm{~K}$. The total absorption coefficients presented in Fig. 3 were calculated with the use of formulas $1-4$ for a pressure of 60 and $10 \mathrm{MPa}$. The calculations were made assuming that the electron temperature $T_{e}$ and the temperature of heavy particles $T_{h}$ were the same. The ratio between absorption coefficients for 1064, 532, and $355 \mathrm{~nm}$ is approximately 9:2:1 for temperatures greater than $15 \mathrm{kK}$.

\section{Experiment}

Graphite target irradiation was performed using a Nd:YAG laser. The laser operated at a wavelength of 1064, 532, $355 \mathrm{~nm}$ with a pulse energy of $\sim 400 \mathrm{~mJ}$ and $10 \mathrm{~ns}$ pulse duration. The laser intensity was $I=1.5 \mathrm{GW} \mathrm{cm}^{-2}(F=$
$15 \mathrm{~J} \mathrm{~cm}^{-2}$ ). The laser spots on the target were $2.5 \mathrm{~mm}^{2}$ in each case. The incident angle of the laser beam was $45^{\circ}$ to the surface normal. A pyrolytic graphite sputtering target was used. The target was rotated to avoid crater formation. The ablated plume expanded in a chamber evacuated to a background pressure of $5 \times 10^{-4} \mathrm{~Pa}$.

The experimental setup was similar to that described in [6]. The emission spectra of the plasma plume were registered with the use of a spectrograph/monochromator (Acton, model SpectraPro2500i) and an ICCD camera. The spectrograph was equipped with three gratings 2400,1800 , and 600 grooves $/ \mathrm{mm}$. In the case of $2400 \mathrm{~g} / \mathrm{mm}$, the reciprocal dispersion in the visible region was $0.005-0.008 \mathrm{~nm} /$ pixel depending on a wavelength. The width of the entrance slit was $50 \mu \mathrm{m}$. The plasma was imaged on the entrance slit using $300 \mathrm{~mm}$ achromatic lens mounted on a movable table. The magnification was 1 . The camera was gated by a digital delay generator triggered by the signal from the laser. The gate width was $10-30 \mathrm{~ns}$ depending on the distance from the target.

The electron densities and temperatures were determined at various distances from the target (from 0.3 to $4 \mathrm{~mm}$ ). At each distance, the delay time was adjusted so as to gather the radiation at the maximum intensity of the spectral line from the highest observed excited level, i.e., C IV $580.13 \mathrm{~nm}$ line, next C III $569.59 \mathrm{~nm}$ line, and at last (in case of second and third laser harmonic) C II $426.7 \mathrm{~nm}$ line. This procedure was chosen to get possibly maximum plasma temperatures. The delay time depended on the distance from the target and was changed from 10 to 85 nanoseconds. The lines used for determination of the electron density and temperature were checked to be free from self-absorption according to the method described in [6].

\section{Results and discussion}

Distinguishable line spectra at early phase of plasma formation could be observed at a distance of $\sim 0.36-0.72 \mathrm{~mm}$ from the target surface, depending on the laser wavelength. Closer to target the line spectra merge to continuum due to high electron density.

In the case of $1064 \mathrm{~nm}$ laser wavelength, the electron densities were determined from the Stark broadening of the C IV $580.13 \mathrm{~nm}$, C III $569.59 \mathrm{~nm}$, and $464.74 \mathrm{~nm}$ lines and at greater distances from the Stark broadening of the C II 426.70/72, 566.24, and $657.80 \mathrm{~nm}$ lines. In the case of 532 and $355 \mathrm{~nm}$, only profiles of the C III and C II lines were used.

Satisfactory signal to noise ratio was achieved after accumulation of 50-150 shots, depending on the spectral line and the distance from the target. The full profile of the measured spectral lines was a Voigt profile resulting from the 
convolution of a Gaussian (apparatus and Doppler) profile and Lorenzian (Stark) profile. The Stark broadening parameters were taken from $[14,15]$. The apparatus half-width was measured with the use of low pressure spectral lamp. The Stark broadening is proportional to electron density, the correction due to ion broadening is less than $10 \%$.

In the case of 1064 laser wavelength, close to the target the electron temperature was determined from the ratio of intensities of the C IV $580.13 \mathrm{~nm}$ and C III $569.59 \mathrm{~nm}$ lines (multiplied by the electron density). Farther from the relative intensities of the C III 466.58, 466.36, 467.39, 464.74, 465.02, 465.14, and $569.59 \mathrm{~nm}$ lines (Boltzmann plot) and from the ratio of intensities of the C III $569.59 \mathrm{~nm}$ and C II $566.24 \mathrm{~nm}$ lines.

It was clear from the observed spectra that the temperature in the case of 532 and $355 \mathrm{~nm}$ laser pulse is lower. Neither C IV lines nor C III 466.58, 466.36, 467.39 nm lines from highly excited levels were observed. Therefore, the temperatures were determined from the ratio of intensities of the C III $569.59 \mathrm{~nm}$ and C II $566.24 \mathrm{~nm}$ lines and, farther from the target from the relative intensities of the C II $387.64,392.07,426.7 / 2,566.20,657.80,658.28,678.39$, $675.08,723.13$, and $723.64 \mathrm{~nm}$ lines. The transition probabilities were taken from [16] and [17].

The results are shown in Figs. 4 and 5. They were obtained from the line profiles integrated over the plasma depth seen by the central track $(1$ track $=130 \mu \mathrm{m})$ of the detector. It has been found that the maximum temperatures and densities derived from the Abel inverted line intensities are only slightly higher in comparison to their integrated values. For the sake of clarity, only some representative results are shown in the figures.

The electron densities as a function of distance from the target are shown in Fig. 4. In the case of $1064 \mathrm{~nm}$ wavelength, the electron density reaches $\sim 5 \times 10^{24} \mathrm{~m}^{-3}$ near the

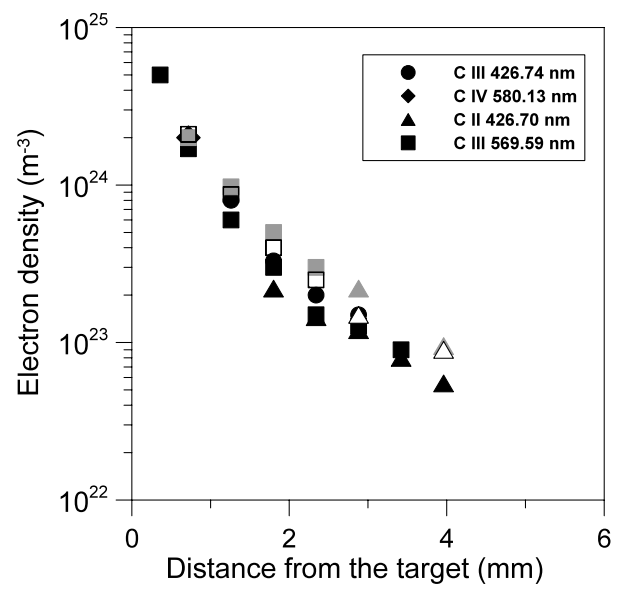

Fig. 4 Electron density as a function of a distance from the target. Black symbols: $1064 \mathrm{~nm}$, gray symbols: $532 \mathrm{~nm}$, open symbols: $355 \mathrm{~nm}$. Delay times $10,15,25,35,45,55,70,85 \mathrm{~ns}$, gate widths $10,15,30 \ldots 30 \mathrm{~ns}$ target and drops to $\sim 5.5 \times 10^{22} \mathrm{~m}^{-3}$ at a distance of $4 \mathrm{~mm}$. In the case of 532 and $355 \mathrm{~nm}$ the electron density could not be determined so close to the target because at distances less than $0.7 \mathrm{~mm}$ all lines merged to continuum. Farther from the target, the electron densities are slightly higher than in the case of $1064 \mathrm{~nm}$ wavelength. No clear differences between $532 \mathrm{~nm}$ and $355 \mathrm{~nm}$ have been found. The accuracy is within $\pm 40-60 \%$ depending on the line which includes accuracy of the experimental value of Stark broadening and the experimental error. Relative errors between various wavelengths are much smaller because they do not include accuracy of the Stark widths.

The electron temperatures are shown in Fig. 5. In the case of $1064 \mathrm{~nm}$, the electron temperature reaches $\sim 68 \mathrm{kK}$ near the target and drops to $\sim 30 \mathrm{kK}$ at a distance of $\sim 4 \mathrm{~mm}$ from the target. The corresponding values for 532 and $355 \mathrm{~nm}$ are much lower. The temperatures are $\sim 44 \mathrm{kK}$ at a distance $0.72 \mathrm{~mm}$ from the target and decreases to $\sim 24 \mathrm{kK}$ at a distance of $\sim 4 \mathrm{~mm}$. Again no clear differences between $532 \mathrm{~nm}$ and $355 \mathrm{~nm}$ are found. The accuracy is $\pm 15 \%$. Since after the cessation of the laser pulse the energy equilibration time between electrons and heavy particles is a few nanoseconds, we can assume that close to the target $T_{e}=T_{a}$.

The observed differences in the electron temperatures do not fit the theoretical predictions. The ratio between absorption coefficients for 1064,532 , and $355 \mathrm{~nm}$ is approximately 9:2:1. Although it explains why for $1064 \mathrm{~nm}$ wavelength the highest temperatures are observed, it does not explain the lack of differences in the case of 532 and $355 \mathrm{~nm}$. In addition, the electron densities are slightly higher in case of shorter wavelengths. These findings can be explained by the effect of enhanced ablation rate in case of shorter wavelengths. Due to change from solid to liquid phase the reflectivity of graphite is similar for 1064 and $532 \mathrm{~nm}$ [18] and we can expect it is similar for $355 \mathrm{~nm}$. On the other hand,

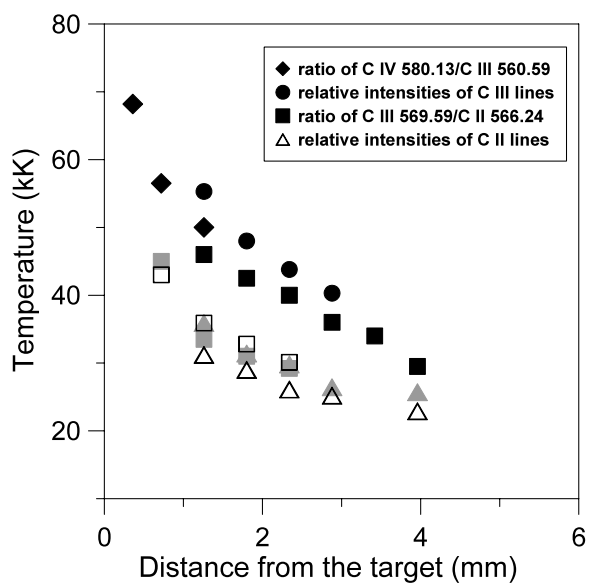

Fig. 5 Electron temperature as a function of distance from the target. Black symbols: $1064 \mathrm{~nm}$, gray symbols: $532 \mathrm{~nm}$, open symbols: $355 \mathrm{~nm}$. Delay times and gate widths as in Fig. 4 
the optical penetration depth is smaller for shorter wavelengths [19]. This means that nearly the same amount of laser energy is coupled to the smaller volume resulting in higher target temperature, and hence in higher initial pressure and temperature of the plume. This effect masks the effect of laser wavelength on plasma heating.

Quicker decrease of $n_{e}$ and $T_{e}$ observed in the case of $1064 \mathrm{~nm}$ can be explained by faster expansion of the hotter plume. The velocities were determined using the time of flight method [6]; the directed forward mass center velocities were $\sim 5 \times 10^{4}$ and $6 \times 10^{4} \mathrm{~m} \mathrm{~s}^{-1}$ in the case of $355 / 532 \mathrm{~nm}$ and $1064 \mathrm{~nm}$, while velocities of the expansion relative to the center were $8 \times 10^{3}$ and $1 \times 10^{4} \mathrm{~m} \mathrm{~s}^{-1}$, respectively.

Unlike the determination of the electron density from the Stark broadening for which only Maxwellian distribution of the electrons is desirable, the determination of the electron temperature requires that the levels considered are in the local thermodynamic equilibrium. The method of assessment of the LTE was similar to that described in [6] and proved that LTE conditions should prevail in the range of the studied plasma parameters. This conclusion is supported by the fact that the temperatures derived from the Boltzmann plots and Saha equation agree within experimental errors.

\section{Summary}

The influence of the laser wavelength on heating of ablated carbon plume was analyzed. It has been found that the temperatures obtained in the experiment cannot be explained simply by the calculated plasma absorption coefficients. The plasma temperatures are highest in the case of $1064 \mathrm{~nm}$ but no clear differences between 532 and $355 \mathrm{~nm}$ have been found though the calculated absorption coefficients show the progressive increase with the laser wavelength. The electron densities are slightly higher in case of 355 and $532 \mathrm{~nm}$. The results can be explained by the effect of enhanced ablation rate in the case of shorter wavelengths.

Acknowledgement This work was supported by the Research Project N N501 069138.
Open Access This article is distributed under the terms of the Creative Commons Attribution Noncommercial License which permits any noncommercial use, distribution, and reproduction in any medium, provided the original author(s) and source are credited.

\section{References}

1. S.S. Harilal, C.V. Bindhu, R.C. Issac, V.P.N. Nampoori, C.P.G. Vallabhan, J. Appl. Phys. 82, 2140 (1997)

2. J. Hermann, C. Vivien, A.P. Carricato, C. Boulmer-Leborgne, Appl. Surf. Sci. 127-129, 645 (1998)

3. Y. Yamagata, A. Sharma, J. Narayan, R.M. Mayo, J.W. Newman, K. Ebihara, J. Appl. Phys. 88, 6861 (2000)

4. P. Loiseleur, T.N. Hansen, J. Larour, J.G. Lunney, Appl. Surf. Sci. 197-198, 164 (2002)

5. H. Luna, J. Dardis, D. Doria, J.T. Costello, Braz. J. Phys. 37, 1301 (2007)

6. J. Hoffman, W. Mróz, A. Prokopiuk, Z. Szymanski, Appl. Phys. A, Mater. Sci. Process. 92, 921 (2008)

7. J. Richter, in Plasma Diagnostics, ed. by A.A. Lochte-Holtgreven (North Holland, Amsterdam, 1968)

8. F. Cabannes, J.C. Chapelle, in Reactions Under Plasma Conditions, ed. by M. Venugopalan (Wiley, New York, 1971)

9. H.R. Griem, Plasma Spectroscopy (McGraw-Hill, New York, 1964)

10. S.N. Nahar, NORAD-Atomic-Data (Nahar_OSU_Radiative_ Atomic_Data); http://www.astronomy.ohio-state.edu/ nahar/ nahar_radiativeatomicdata/index.html

11. L.D. Thomas, R.K. Nesbet, Phys. Rev. A 12, 2378 (1975)

12. C.J. Knight, AIAA J. 17, 519 (1979)

13. N.M. Bulgakova, A. Bulgakov, L.P. Babich, Appl. Phys. A, Mater. Sci. Process. 79, 1323 (2004)

14. N. Konjevic, A. Lesage, J.R. Fuhr, W.L. Wiese, J. Phys. Chem. Ref. Data 31, 819 (2002)

15. N. Konjevic, W.L. Wiese, J. Phys. Chem. Ref. Data 19, 1307 (1990)

16. Yu. Ralchenko, A.E. Kramida, J. Reader, NIST ASD Team, NIST Atomic Spectra Database (ver. 4.0.0). National Institute of Standards and Technology, Gaithersburg, MD (2010)

17. R.L. Kurucz, B. Bell, Atomic Line Data, Kurucz CD-ROM No. 23. Smithsonian Astrophysical Observatory, Cambridge (1995)

18. A.M. Malvezzi, N. Bloembergen, C.Y. Huang, Phys. Rev. Lett. 57, 146 (1986)

19. N.M. Bulgakova, A. Bulgakov, O.L. Bobrenok, Phys. Rev. E 62, 5624 (2000) 